\title{
Synthesis and Corrosion Inhibition Application of some New Schiff Bases Based on 1,3-diaminopropane
}

\author{
M.E.Moustafa, A.I.Ali, A.Y.El-Etre and H.A.Ibrahim \\ Chemistry Dept., Faculty of Science, Benha Univ,Benha,Egypt \\ E-Mail: hend.hegazy89@gmail.com
}

\begin{abstract}
Synthesis and characterization of a new Schiff base ligand expected to have ligating and corrosion inhibition properties are performed. The organic ligand was synthesized from the condensation of $\mathrm{p}$ Chlorobenzaldehyde and 1,3-diaminopropane in ethanol [2:1 molar ratio respectively $\}$ and its chemical structure was investigated by different physical and spectroscopic techniques [elemental analysis, FTIR, electronic absorption spectra and ${ }^{1} \mathrm{H}$ NMR spectra]. A group of measurements involving $\mathrm{DMOL}^{3}$ program in materials studio package which is shaped for the recognized of wide scale Density Function Theory [DFT] were applied. The molecular modeling and total density using DFT method for the compound were determined and given. The newly prepared Schiff base was tested as acorrosion inhibitor for carbon steel in $\mathrm{HCl}$ solution.
\end{abstract}

Keywords: 1,3-diaminopropane Schiff base, Spectral analysis, Molecular modeling, Corrosion inhibitor.

\section{Introduction}

Schiff's bases are an important class of organic compounds which have broad ranges of biological activities including antitumor [1-3], anticancer [46], antibacterial [7-9], antifungal [10] and diuretic [11] activity. Those of salicylaldehyde derivatives had been reported as plant growth regulators [12] and antimicrobian [13] or antimycotic activities [14]. They are characterized by the $-\mathrm{N}=\mathrm{CH}-$ [imine] group which imports in elucidating the mechanism of transamination and rasemination reaction in biological system [15-17]. They are used as catalysts in medicine, such as in antibiotics and antiinflammatory agents, and in industry as an anticorrosive [18-20] and also as analytical reagents for spectrophotometric metal analysis [21, 22]. The great interest in Schiff bases derived from diamines is due to their ability to act as bidentate ligands for transition metal ions [23, 24]. In recent years, computational chemical models are playing an ever increasing role in chemical research. HF and DFT methods are the common used methods in many reported references [25, 26]. Among DFT calculation, Becke's three parameter hybrids functional combined with the Lee-Yang-Parr correlation functional [B3LYP] is the best predicting results for vibrational wave numbers for moderately lager molecule $[27,28]$. Moreover, it is known that the DFT [B3LYP] method adequately takes into account electron correlation contributions, which are especially in systems containing extensive electron conjugation and/or electron lone pairs [29]. The present work aims to study the synthesis, characterization and structure elucidation of one Schiff base ligand prepared from condensation of $\mathrm{p}$ - Chlorobenzaldehyde and 1,3-diaminopropane in ethanol [2:1 molar ratio respectively]. The newly prepared Schiff base was tested as corrosion inhibitor for carbon steel in $\mathrm{HCl}$ solution. Many researches were conducted to investigate the Schiff base compounds as inhibitors for steel corrosion [30 - 33]. In this study, the newly prepared Schiff base was tested as corrosion inhibitor for carbon steel in $\mathrm{HCl}$ solution. Weight loss measurements, potentiodynamic polarization and electrochemical impedance spectroscopy techniques were used for corrosion rate measurements.

\section{Experimental \\ 2.1Materials}

All reagents and solvents used in the present study were of the highest quality [Merck, Aldrich, Fluka or Sigma Research Laboratories] and were used without further purification. 1,3diaminopropane was of purity $98 \%$ and was used without further purification.

\subsection{Preparation of the Schiff base}

The new Schiff base was prepared by condensation of 1,3-diaminopropane $[1.00 \mathrm{mmol}$ in ethanol] with $\mathrm{p}$ - Chlorobenzaldehyde [2.00 mmol]. A hot ethanolic solution [1.00 mmol] of the diamine was added with stirring to another hot ethanolic solution of $2.00 \mathrm{mmol}$ of $\mathrm{p}$ Chlorobenzaldehyde and the mixture was refluxed for $\approx 3$ hours, then the yellow precipitate was filtered off, washed thoroughly with hot distilled water, dried in vacuum and recrystallized from ethanol.

\subsection{Physical measurements}

Carbon, hydrogen and nitrogen $[\mathrm{C}, \mathrm{H}$ and $\mathrm{N}]$ of the free Schiff base was determined by elemental analysis at the microanalytical Centre, Cairo 
University, Giza, Egypt. FTIR spectra were performed as $\mathrm{KBr}$ disc technique. Percent transmittance was recorded against wavenumber $\left[\mathrm{cm}^{-1}\right]$ using FTIR spectrometer Model Nicolet is 10-thermo-scientific with the wavenumber range $4000-400 \mathrm{~cm}^{-1}$ carried out at Faculty of Science, Benha University. Electronic absorption spectra were recorded on Jasco V - 530 [UV-Vis] double beam spectrophotometer [Japan] with scanning speed $400 \mathrm{~nm} / \mathrm{min}$ and band width $2.0 \mathrm{~nm}$ using 10 $\mathrm{mm}$ matched quartz cell at room temperature in the range $800-200 \mathrm{~nm}$ at Faculty of Science, Benha University, Benha, Egypt. The Cluster calculations using $\mathrm{DMOL}^{3}$ program [34] in materials studio package [35] which is designed for the realize density functional theory calculation [DFT] were performed. DFT semi-core pseudopods calculations [dspp] were performed with the double numerical basis sets plus polarization functional [DNP]. The DNP basic sets are of comparable quality to 6-31 G Gaussian basis sets [36].

\subsection{Experimental Methods for electrochemical studies}

\subsubsection{Materials}

Carbon steel [C45] with composition [[Fe], C [0.42-0.50\%], Si $[<0.40 \%], \mathrm{Mn}[0.5-0.8 \%], \mathrm{P}[<$ $0.045 \%], \mathrm{S}[<0.045 \%], \mathrm{Cr}[<0.4 \%], \mathrm{Mo}[<0.1$ $\%$ ], $\mathrm{Ni}[0.4 \%],-\{\mathrm{Cr}+\mathrm{Mo}+\mathrm{Ni}=<0.63 \%\}]$ was used. Pure cupper $99.99 \%$ was used as a test metal. Rectangular sheets of both metals with dimensions of $1 \times 1 \mathrm{~cm}$ were used in gravimetric experiments. For electrochemical techniques, a rod with cross-section area of $1 \mathrm{~cm}^{2}$ was used. The rod was embedded in Araldite leaving the bottom area exposed to the aggressive medium. A pretreatment procedure was carried out, prior to each experiment, in which the surface of specimen was mechanically polished with different emery papers, cleaned with acetone and distilled water then dried at room temperature before use.

\subsubsection{Preparation of the solutions}

The aggressive solutions; a stock solution of 2 $\mathrm{M}$ hydrochloric acid [37\%] with density of 1.185 $\mathrm{g} / \mathrm{cm} 3$ was prepared using distilled water. The desired acid concentrations were prepared by diluting the appropriate volume of the stock solution acid with distilled water. The concentration of acid was checked by titration of appropriately diluted portion with standard solution of sodium carbonate.

\subsection{Experimental techniques}

\subsubsection{Weight loss method}

Prior to each experiment the surface of carbon steel specimens were mechanically polished with different grades of emery papers and rinsed by bidistilled water and dried between two filter papers. The cleaned carbon steel was weighed before and after immersion in $50 \mathrm{ml}$ of the test solution for a period up to $24 \mathrm{hr}$. The average weight loss for each two identical experiments was taken and expressed in $\mathrm{mg} / \mathrm{cm}^{2}$. The temperature was adjusted to $25 \pm 1{ }^{\circ} \mathrm{C}$ using a thermostat. Inhibition efficiencies were calculated from the weight loss data using the relation:

$$
I E \%=\left[\frac{W_{f}-W_{i}}{W_{f}}\right] x 100
$$

Where IE\% is the percentage Inhibition efficiency, $\mathrm{W}_{\mathrm{f}}$ and $\mathrm{W}_{\mathrm{i}}$ are the weight loss in absence and presence of inhibitors, respectively.

\subsubsection{Potentiodynamic polarization}

Potentiodynamic polarization method was used to determine the anodic and cathodic polarization curves of carbon steel in $1 \mathrm{M} \mathrm{HCl}$ in absence and presence of different concentrations of inhibitors. E vs. log I curves were recorded using Metrohm potentiostat supported with Nova software for calculations. The corrosion kinetic parameters such as corrosion current [Icorr], corrosion potential [Ecorr], cathodic Tafel slope $[\beta c]$, and anodic Tafel slope $[\beta a]$ were derived from the Tafel curves.

The corrosion rate was calculated by polarization method as the current corresponds to the intercept between cathodic and anodic lines. Inhibition efficiency was calculated from the equation:

$$
I E \%=\left[\frac{r_{f}-r_{i}}{r_{f}}\right] x 100
$$

Where $r_{f}$ and $r_{i}$ are the corrosion rates in absence and presence of the extract.

\subsubsection{Electrochemical impedance spectroscopy [EIS]}

The EIS spectra were recorded at open circuit potential, OCP after immersion the electrode for $30 \mathrm{~min}$. The AC signal was $5 \mathrm{mV}$ peak to peak and the frequency range studied was between $50 \mathrm{kHz}$ and $0.1 \mathrm{~Hz}$ by using same potentiostat as in potentiodynamic polarization.

Inhibition efficiency was calculated from the equation:

$$
I E \%=\left[\frac{R_{c t i}-R_{c t f}}{R_{c t i}}\right] x 100
$$


Where $\mathrm{R}_{\mathrm{ctf}}$ and $\mathrm{R}_{\mathrm{cti}}$ are the corrosion rates in absence and presence of the extract.

The polarization experiments were carried out using three-apartment electrochemical cell with platinum foil counter electrode and saturated calomel electrode as reference electrode.

\section{Results and Discussion}

\section{A- $\quad$ Synthesis and characterization of the newly prepared Schiff base}

The Schiff base under study was synthesized by the usual rout for preparing such compounds. 1,3-diaminopropane undergoes condensation reaction with $\mathrm{p}-$ Chloro-benzaldehyde as shown in the following Scheme:

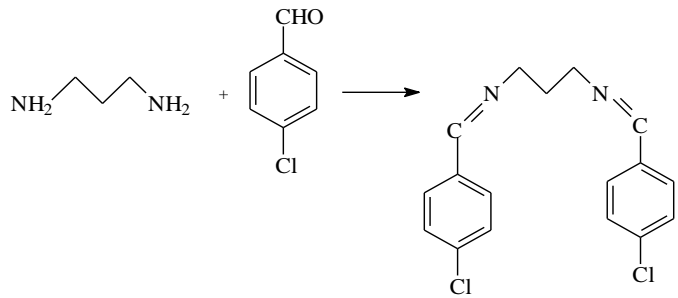

The Schiff base proposed formula was ascertained by the following techniques:

\section{- Elemental analysis}

The elemental analysis of the compound under study show satisfactory agreement with the proposed formula. Tentative formula; $\mathrm{C}_{17} \mathrm{H}_{16} \mathrm{Cl}_{2} \mathrm{~N}_{2}$, M. Wt. 319.23, color; pale yellow, M.P. $120-122^{\circ} \mathrm{C}, \% \mathrm{C} 63.96$ [found 63.21], $\% \mathrm{H}$ 5.05 [found 4.89], $\% \mathrm{~N} 8.78$ [found 8.34] and $\% \mathrm{Cl}$ 22.21 [found 21.34].

\section{- FTIR Spectra}

A satisfactory band assignment for the IR spectrum of the Schiff base under study was done (c.f. Fig (1). The assignment of the band frequencies was carried out by applying a method similar to that suggested by Looker [37] according to which the spectrum was subdivided into four regions, namely; the $4000-2800,1700-1500$, $1500-1000$ and $1000-625 \mathrm{~cm}^{-1}$. The band frequencies $\left[\mathrm{cm}^{-1}\right]$ of the most important characteristic functional groups are summarized as follows

- In the range $2888-2856 \mathrm{~cm}^{-1}$, the IR spectrum reveal two absorption bands assigned to the symmetric and asymmetric stretching vibrations of the $-\mathrm{CH}_{2}$ - group in aliphatic chain.

- In the frequency range $1800-1200 \mathrm{~cm}^{-1}$, the most important bands appearing are due to vibrations of the $\mathrm{C}=\mathrm{C}$ aromatic, $\mathrm{C}=\mathrm{N}$ and $\mathrm{C}=\mathrm{O}$ bonds. In our case, the strong band appearing at $1620 \mathrm{~cm}^{-1}$ is due to the symmetric stretching vibrations of the $\mathrm{C}=\mathrm{N}$ group.

- Most of the strong bands appearing in the range $1000-500 \mathrm{~cm}^{-1}$ are due to the bending C-H outof-plane deformation vibrations present in the aromatic moieties. The band positions can be discussed in terms of number of adjacent hydrogen atoms. These bands give important means for determining the nature of aromatic substitution. The medium band at $760 \mathrm{~cm}^{-1}$ in the spectrum of the compound under study corresponds to the bending deformation of vibration of two adjacent hydrogen atoms.

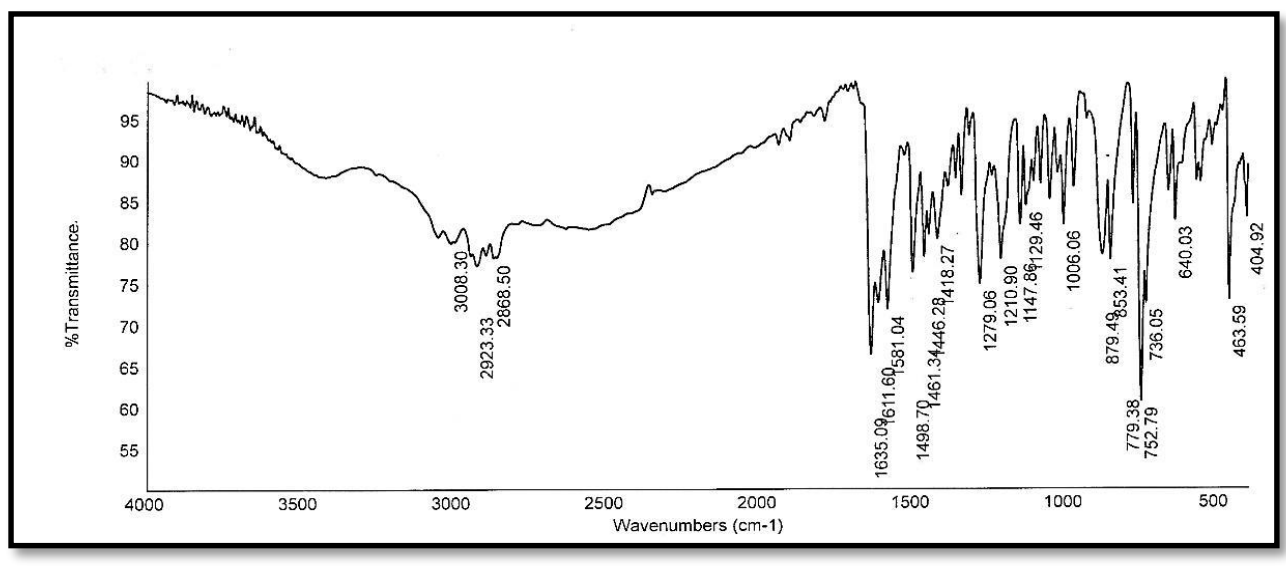

Fig (1) IR spectrum of the Schiff base [I]

\section{${ }^{1} \mathrm{H}$ NMR spectra}

An important tool for the elucidation of chemical structure is the ${ }^{1} \mathrm{H}$ NMR spectrum in DMSO as a solvent. The technique becomes more helpful when measurements are performed after deuteration with $\mathrm{D}_{2} \mathrm{O}$ where replaceable protons are removed, consequently the type of hydrogen atom is accurately confirmed. To get successful 
assignment of the spectra, similar types of hydrogen atoms are classified into group. This can be done, simply by numeration of each type of protons according to their position in the molecule. In the present study, numeration of hydrogen atoms is as shown in the following schemes:

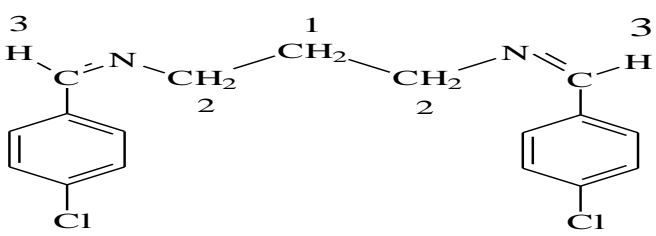

Hydrogen numeration of compound I

The chemical shifts $[\delta ; \mathrm{ppm}]$ for the different protons in the Schiff bases I - V are listed in Table [1] and the 1H NMR spectrum is shown in Fig [2-a and $\mathrm{b}]$.

Table (1) Chemical shifts [ $\delta$; ppm] for the different protons in the Schiff base I

\begin{tabular}{ccccccc}
\hline Compound & $\mathbf{X}$ & $\mathbf{H}[1]$ & $\mathbf{H}[2]$ & $\mathbf{H}[3]$ & $\mathbf{X}$ & Aromatic protons \\
\hline $\mathrm{I}$ & $\mathrm{Cl}$ & 2.025 & 3.684 & & - & 8.583 \\
\hline
\end{tabular}

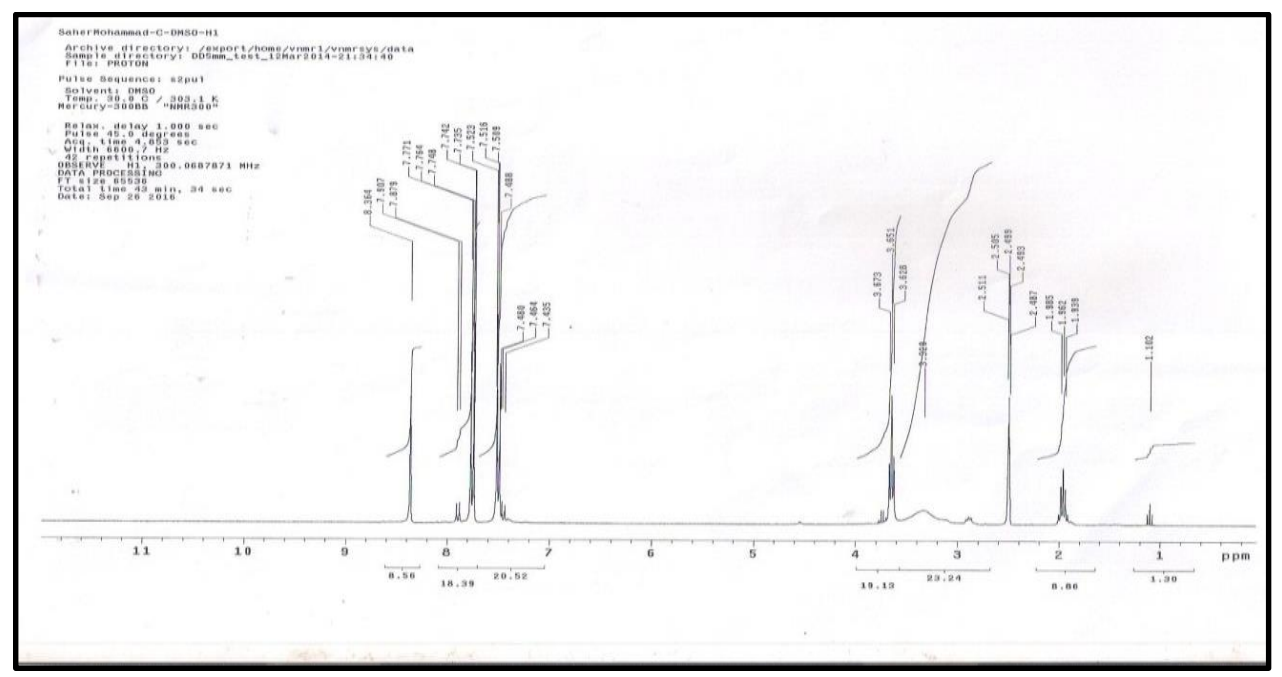

Fig (2-a) ${ }^{1}$ HNMR spectrum of compound I in DMSO

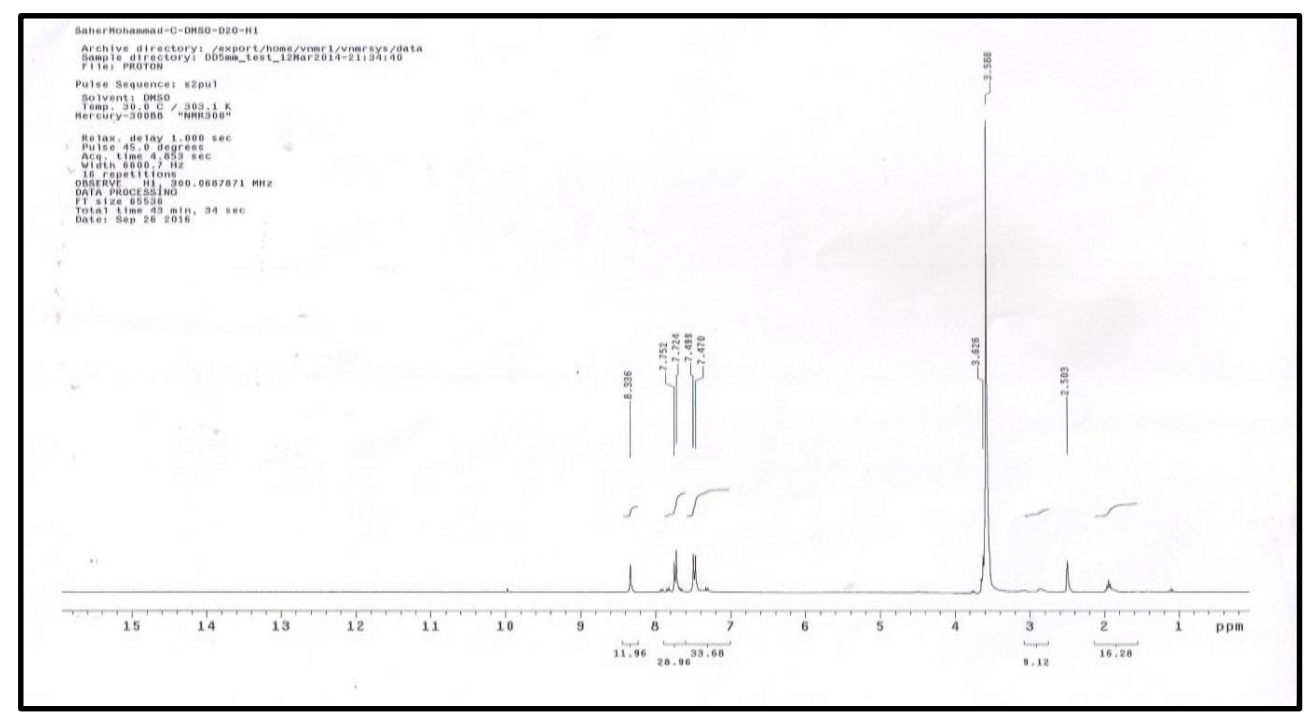




\section{Electronic absorption spectra}

Fig (2-b) ${ }^{1} \mathrm{HNMR}$ spectrum of compound I in DMSO-D ${ }_{2} \mathrm{O}$

The present study is devoted to investigating the UV - visible spectra in ethanol and in different organic solvents of varying polarities and band assignment in relation to molecular structure. The spectra were recorded in ethanol [polar solvent] and cyclohexane [nonpolar solvent] in the range $190-600 \mathrm{~nm}$. The spectral data are cited in Table (2). In ethanol, the spectrum display mainly four absorption bands: the first two bands $[\mathrm{A}]$ and $[\mathrm{B}]$ within the range $214-233 \mathrm{~nm}$ and $230-340 \mathrm{~nm}$ are due to the medium and low energy $\pi-\pi^{*}$ transitions within the aromatic moieties representing the $\left[{ }^{1} \mathrm{~L}_{\mathrm{a}} \rightarrow 1_{\mathrm{A}}\right]$ and $\left[{ }^{1} \mathrm{~L}_{\mathrm{b}} \rightarrow 1_{\mathrm{A}}\right]$ states, respectively [38]. This is confirmed by the high value of molar extinction coefficient $\left[\varepsilon ; \mathrm{dm}^{3} \mathrm{~mol}^{-}\right.$ ${ }^{1} \mathrm{~cm}^{-1}$ ], c.f.Table (2). The third band [C] appearing within the range $306-337 \mathrm{~nm}$ is due to the $n \rightarrow \pi^{*}$ transitions in the $\mathrm{C}=\mathrm{N}$. The fourth band $[\mathrm{D}]\left[\lambda_{\max }\right.$ within the range $358-420 \mathrm{~nm}$ ] can be assigned to the charge transfer $[\mathrm{CT}]$ transition through the whole molecule represented as

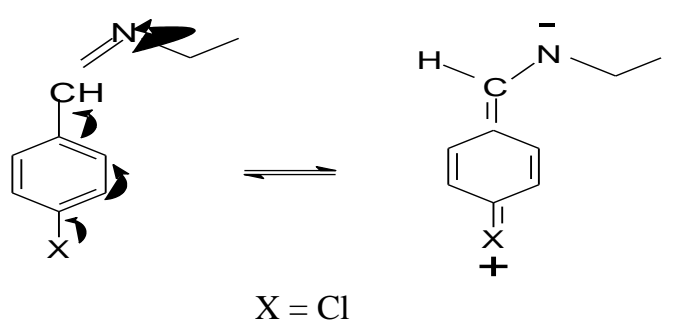

The spectrum in cyclohexane show also four absorption band. The first two are assigned, as discussed before, to the medium and low energy $\pi$ $\pi^{*}$ transitions within the aromatic moieties representing the $\left[{ }^{1} \mathrm{~L}_{\mathrm{a}} \rightarrow 1_{\mathrm{A}}\right]$ and $\left[{ }^{1} \mathrm{~L}_{\mathrm{b}} \rightarrow 1_{\mathrm{A}}\right]$ states, respectively while the third and fourth bands represent the $n-\pi^{*}$ transition and the charge transfer interaction through the whole molecule. The values of molar extinction coefficient $[\varepsilon]$ were not calculated because the spectra were recorded for saturated solutions due to insolubility in cyclohexane.

Table (2) UV-Vis spectral bands of the Schiff base [I] in ethanol and cyclohexane

\begin{tabular}{|c|c|c|c|c|c|c|c|c|}
\hline \multirow[t]{2}{*}{ Solvent } & \multicolumn{2}{|c|}{$\mathbf{A}$} & \multicolumn{2}{|c|}{ B } & \multicolumn{2}{|c|}{$\mathbf{C}$} & \multicolumn{2}{|c|}{$\mathbf{D}$} \\
\hline & $\max \lambda$ & $\max \varepsilon$ & $\max \lambda$ & $\max \varepsilon$ & $\max \lambda$ & $\max \varepsilon$ & $\max \lambda$ & $\max \varepsilon$ \\
\hline Ethanol & 221 & 1.425 & 261 & 8.44 & 323 & 3.01 & 411 & 0.36 \\
\hline cyclohexane & 221 & S.S & 254 & S.S & 286 [sh] & S.S & 296 & S.S \\
\hline
\end{tabular}

\section{Electronic spectra in different solvents}

The absorption spectra of the Schiff base under study were scanned in different solvents of varying polarities [methanol, acetone, n-hexane, dimethylformamide, carbontetrachloride and 1,4dioxane], the spectra are shown in Fig (3). Inspection of the spectra obtained shows that the $\pi-\pi^{*}$ transitions in the UV region display an irregular shift on changing solvent polarity. This behavior is characteristic of the $\pi-\pi^{*}$ transition of substituted benzenes where the solvent substituent interaction would be additive or counteracts the solvent shift [39]. On the other hand, the longer wavelength band corresponding to the intramolecular CT interaction displays some shifts with varying solvent polarity.

To assess the influence of solvents on the position of $\lambda_{\max }$ of the CT band, the so-called macroscopic and microscopic solvent polarity parameters $[\mathrm{f}[\mathrm{D}], \varphi[\mathrm{D}], \pi, \alpha, \beta, \mathrm{ET}$ and Z] are applied [40]. On plotting the value of $\lambda_{\max }$ of the CT band of each compound against each of these parameters, nonlinear relations were obtained. This indicates that none of such parameters solely is the predominating factor affecting the position of the CT band, but the contribution of specific solute - solvent interaction [salvation or hydrogen bonding between solvent and solute] also takes place.

\section{Molecular orbital calculations}

Molecular modeling of the free ligand

A group of measurements involving $\mathrm{DMOL}^{3}$ program in materials studio package which is shaped for the recognized of wide scale Density Function Theory [DFT] were applied. The molecular modeling and total density using DFT method for the compound I were determined and given in structures (1 and 2). 


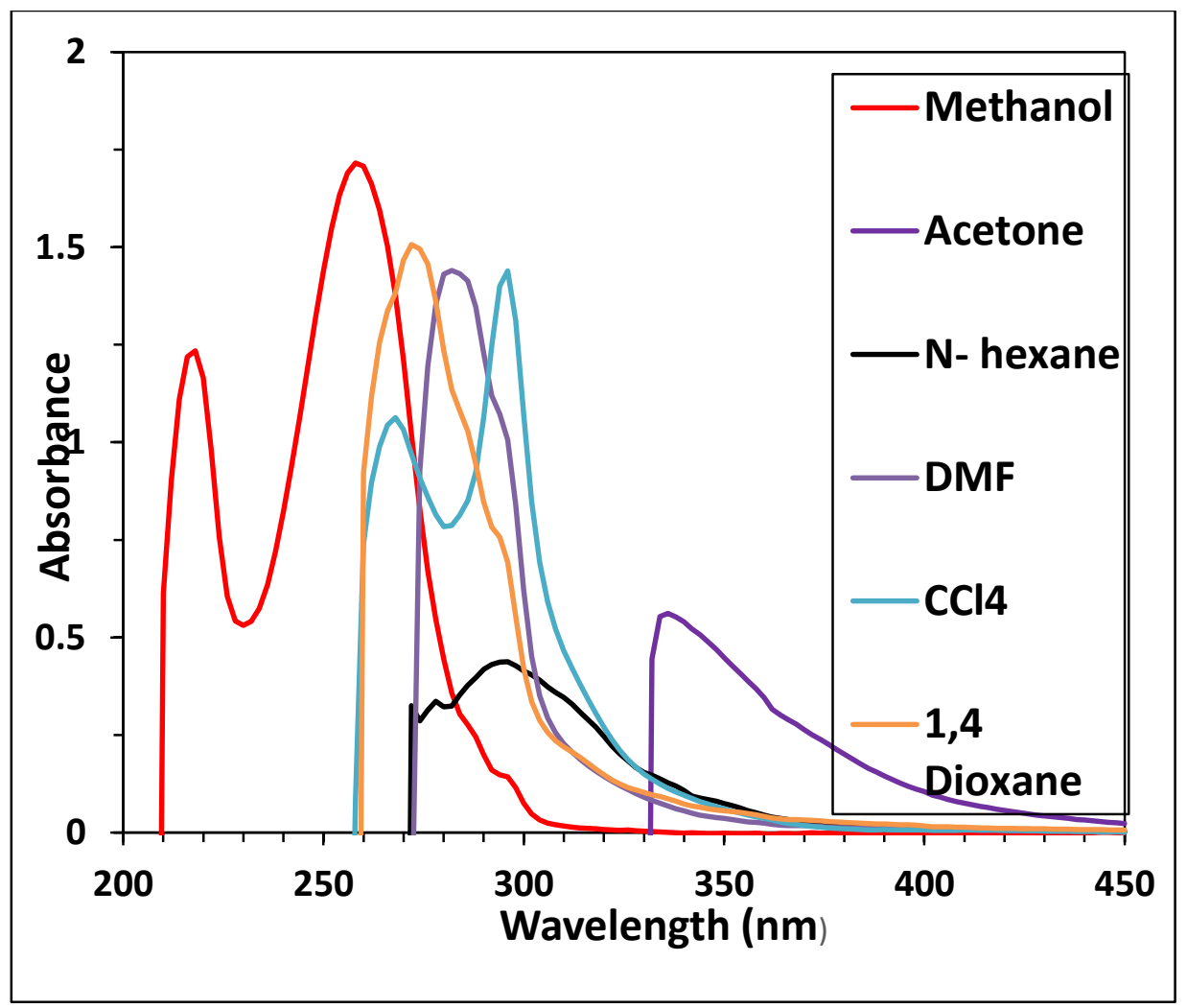

Fig (3) Electronic absorption spectrum of ligand I in different organic solvents

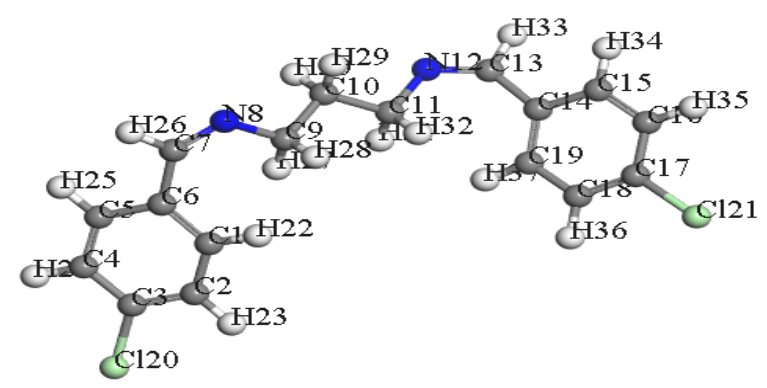

Sch (1) Molecular modeling DFT method for ligand I

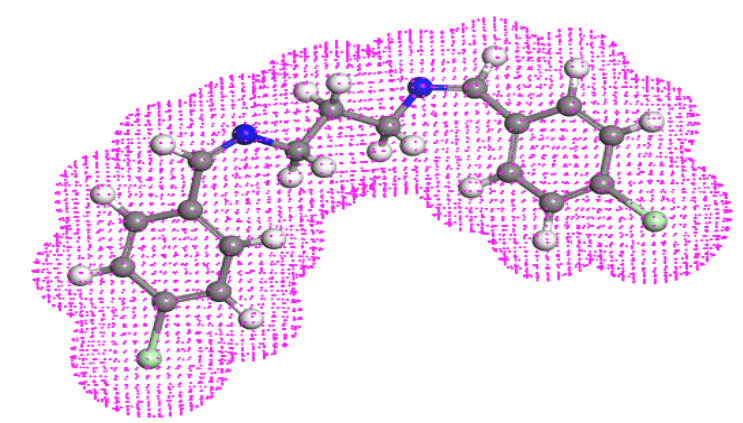

Sch (2) Total density using DFT method for ligand I 


\section{Optimization geometry}

The gas phase geometries of compound I was fully optimized with respect to the energy. For this compound, only the ground state geometries are optimized in accordance with the optimized structure of compounds as shown in structure 1 and the following scheme:

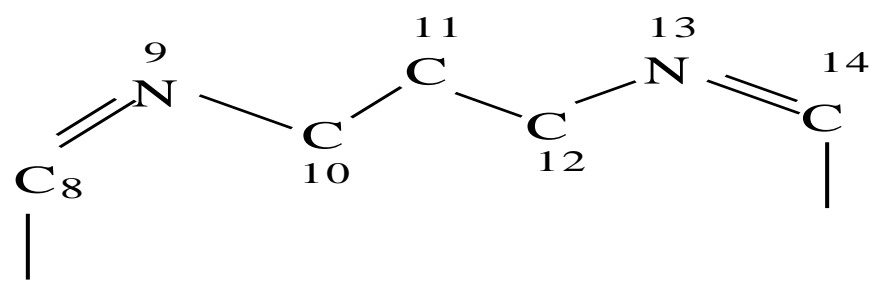

All the bond lengths and bond angles in phenyl rings are in the common range. Relevant bond distances and angles are calculated which are in good agreement with those reported in similar Schiff base compounds [41]. The C8 = N9 and $\mathrm{C} 14=\mathrm{N} 13$ bond lengths are 1.287 and $1.288 \AA$, respectively which are comparable to the imine double bond found in the literature $[\langle\mathrm{C}=\mathrm{N}\rangle \sim 1.27$ $\AA$ ] [42]. The N9 - C10 and C12 - N13 bond lengths are 1.464 and $1.466 \AA$, respectively while those between $\mathrm{C} 10-\mathrm{C} 11$ and $\mathrm{C} 11-\mathrm{C} 12$ are
1.536 and $1.539 \AA$, respectively which are in good agreement with the mean values for a $\mathrm{C}-\mathrm{C}$ and $\mathrm{C}$ $\mathrm{N}$ single bonds [42]. The $\mathrm{C} 8-\mathrm{N} 9-\mathrm{C} 10$ torsion angles of $121.388^{\circ}$ and that between $\mathrm{C} 12-\mathrm{N} 13-\mathrm{C} 14$ $120.559 \mathrm{~A}^{\circ}$ also those between N9-C10-C11 $\left[\begin{array}{ll}110.338 & \AA\end{array}\right]$ and C11-C12-N13 [110.405 $\left.\mathrm{A}\right]$ indicate an almost planar configuration with respect to the imine $\mathrm{C}=\mathrm{N}$ bond [42] and the molecules belong to the $\mathrm{C}_{2 v}$ point group with element of symmetry; $\mathrm{C}_{2}, 2 \sigma_{v}$ [one of them coincide with the $\mathrm{C}_{2}$ axis] and $E$.

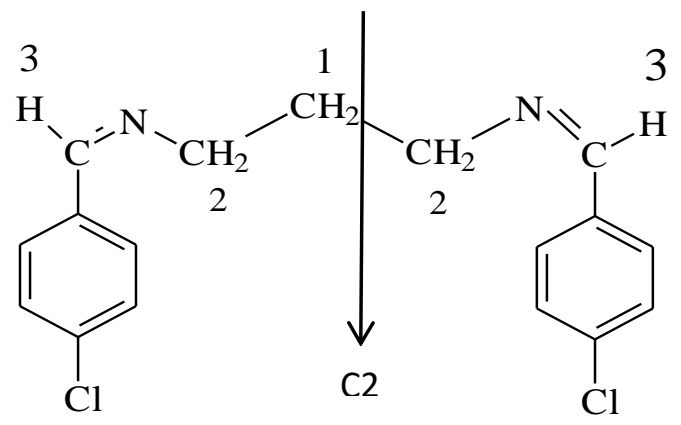

\section{Frontier molecular orbitals}

The HOMOs and LUMOs are known as Frontier molecular orbitals [FMOs], which played an important role for evaluating molecular chemical stability, chemical reactivity and hardness-softness of the molecule [43]. The HOMO and LUMO energy, energy gap $[\Delta \mathrm{E}]$, absolute electronegativity $[\chi]$ Chemical hardness $[\eta]$, softness $[\sigma]$ and electrophilicity index $[\omega][44$, 45] values are listed in Table (3). Picture of Frontier molecular orbitals and Deformation density are shown in Fig. [4]. The HOMO acts as an electron donor, while the LUMO is an electron acceptor. In the HOMO of all selected compounds; the electron density mainly delocalized over associate substituted - saliysaldhyde ring and azomethine group. While in the LUMO orbital this density is delocalized on the azomethine group and the two phenyl rings. The energy gap $[\Delta \mathrm{E}]$ represents the chemical reactivity of compounds. For a system lower value of $\Delta \mathrm{E}$ makes it more reactive or less stable. As depicted in Table (3).

The energy gap $\Delta \mathrm{E}$ is directly involved with hardness/softness of a chemical species. The higher value of $\Delta \mathrm{E}$ represents more hardness or less softness of a compound. Soft molecules are more reactive than hard ones because they could easily offer electrons to an acceptor. Thus, compound I referred as hard molecule when compared to other compounds [46]. Another global reactivity descriptor electrophilicity index $[\omega]$ describes the electron accepting ability of the systems quite similar to hardness and chemical potential. High values of electrophilicity index increases electron accepting abilities of the molecules. 


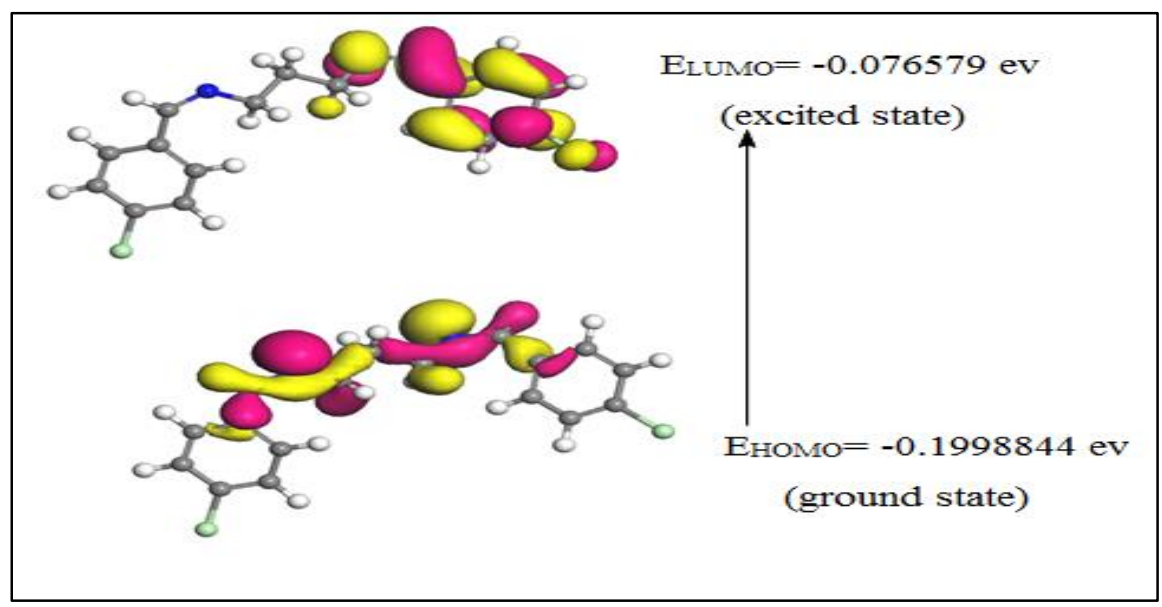

Fig (4) 3D plots frontier orbital energies using DFT method for ligand I

The electrophilicity index $[\omega]$ is positive, definite quantity and the direction of the charge transfer is completely determined by the electronic chemical potential $[\mu]$ of the molecule because an electrophile is a chemical species capable of accepting electrons from the environment and its energy must decrease upon accepting electroic charge. Therefore, the electronic chemical potential must be negative exactly as supported by the values in Table [3]. The values of [Е $\mathrm{HOMO}^{-}$ $\mathrm{E}_{\text {Lомо] }}$ energies of frontier molecular orbitals, energy band gap that explains the final charge transfer interaction inside the molecule, electronegativity $[\chi]$, chemical potential $[\mu]$, global hardness $[\eta]$, global softness $[\sigma]$, additional electronic charge $\left[\Delta \mathrm{N}_{\max }\right]$ and global electrophilicity index $[\omega]$ were calculated and listed in Table [3]:

$$
\begin{array}{ll}
\chi=-1 / 2\left[\mathrm{E}_{\text {LUMO }}+\mathrm{E}_{\text {Hомо }}\right] & \mu=-\chi=1 / 2 \\
{\left[\mathrm{E}_{\text {LUMO }}+\mathrm{E}_{\text {Hомо }}\right]} & \\
\eta=1 / 2\left[\mathrm{E}_{\text {LUмO }}-\mathrm{E}_{\text {Hомо }}\right] & \Delta \mathrm{N}_{\max }=-\mu / \eta \\
\omega=\mu^{2} / 2 \eta &
\end{array}
$$

The inverse value of the global hardness is designed as the softness $[\sigma] ; \sigma=1 / \eta$.

Table (3) The calculated quantum chemical parameters of compound I.

\begin{tabular}{lllllllll}
\hline -HOMO & -LUMO & $\Delta \mathbf{E}$ & $\boldsymbol{\eta}$ & $\boldsymbol{\sigma}$ & $\boldsymbol{\alpha}$ & $\boldsymbol{- \mu}$ & $\boldsymbol{\Omega}$ & $\boldsymbol{\Delta}$ Nmax \\
\hline 0.199884 & 0.07658 & 0.12330 & 0.06165 & 16.2198 & 0.13823 & 0.1382 & 0.15496 & 2.24210 \\
\hline
\end{tabular}

\section{Corrosion measurements}

\section{Weight-loss measurements}

Weight loss measurements were carried out for carbon steel corrosion in $1.00 \mathrm{M} \mathrm{HCl}$ solutions free of and containing different concentrations of compound $[\mathrm{I}]$ at different exposure times. The corrosion rate $[\mathrm{r}]$ was expressed in $\mathrm{g} / \mathrm{cm}^{2}$.day and the obtained data are represented in Table (4). The data of the table show two different behaviors of the tested compound regarding the corrosion of carbon steel. At very small concentrations of the added compound [up to $10^{-4} \mathrm{M}$ ], the presence of the compound enhances the corrosion process of the steel. On the other hand, increasing the added compound over than $10^{-4} \mathrm{M}$ shows the inhibiting effect of the compound toward acid steel corrosion. The corrosive action decreases with increasing the compound concentration, in the lower concentration range, while the inhibition efficiency increases in the higher concentration range. Deep investigation of the data of table (4) reveals that both the opposite effects which have been observed increases with increasing the exposure time of steel to the test medium.

The obtained results could be interpreted in view of the nature of interaction between the additive molecules with the dissolved ferrous cations. The first step of the inhibition process is adsorption of the inhibitor molecules on the corroded metal surface. As the molecule arrives the double layer vicinity it interacts with the dissolved ferric cations forming a complex compound through the complexing center present in its structure. The solubility product of the formed complex is relatively high. Thus, at the low concentration the complex is soluble, and its formation leads to acceleration of the corrosion process. Increasing the additive concentration leads to increasing of the formed complex concentration to above its solubility product and 
consequently it precipitates. The precipitation of the complex on the metal surface form a protection layer which prevent further corrosion of the metal. Further increasing of the additive concentration increasing the formed complex and thus increasing the surface area covered by it. This process leads to an increase of the inhibition efficiency with increasing additive concentration.

Table (4) Weight loss data of Carbon Steel corrosion in free and inhibited 1.00 M HCl solutions of compound [I].

\begin{tabular}{ccccccccc}
\hline \multirow{2}{*}{$\begin{array}{c}\text { Conc. } \\
{[\mathbf{M}]}\end{array}$} & \multicolumn{2}{c}{$\mathbf{1}$ day } & \multicolumn{2}{c}{$\mathbf{2}$ days } & \multicolumn{2}{c}{ 3 days } & \multicolumn{2}{c}{ 4 days } \\
\cline { 2 - 9 } & $\mathbf{r x 1 0}$ & $\mathbf{I E} \%$ & $\mathbf{r x 1 0}^{\mathbf{5}}$ & $\mathbf{I . E \%}$ & $\mathbf{r x 1 0}^{\mathbf{5}}$ & I.E\% & $\mathbf{r x 1 0}^{\mathbf{5}}$ & I.E\% \\
\hline Free & 100 & - & 75 & - & 76 & - & 115 & - \\
$1 \times 10^{-5}$ & 420 & -324 & 320 & -333 & 330 & -342 & 520 & -360 \\
$5 \times 10^{-5}$ & 340 & -248 & 266 & -255 & 270 & -261 & 420 & -269 \\
$1 \times 10^{-4}$ & 102 & -2 & 770 & -3 & 79 & -4 & 120 & -5 \\
$5 \times 10^{-4}$ & 17 & 83 & 180 & 76 & 22 & 71.0 & 39 & 66 \\
$1 \times 10^{-3}$ & 15 & 85 & 135 & 82 & 18 & 76.0 & 36 & 69 \\
\hline
\end{tabular}

\section{Polarization measurement}

Anodic and cathodic curves were traced for polarization of carbon steel electrode in free and inhibited $\mathrm{HCl}$ solutions. The obtained curves are represented in Fig (5). Inspection of the figure reveals that the curves shift toward less current and less negative potential. This observation suggests the inhibitory action of the additive. The calculated corrosion parameters obtained from the polarization curves, by extrapolation method, are presented in Table (5). The data of the table show that the corrosion current increases in presence of low additive concentrations above that of free acid reflecting their corrosivity effect. On the other hand, further increasing of the additive concentration decreases the corrosion rate reflecting inhibition action. This result is consistent with that obtained from weight loss measurements. Moreover, the values of corrosion potential, anodic and cathodic Tafel constants change slightly in the presence of the additive. These results suggest that the additive acts as mixed type inhibitor. This means that the additive molecules adsorb at both anodic and cathodic sites of the steel surface.

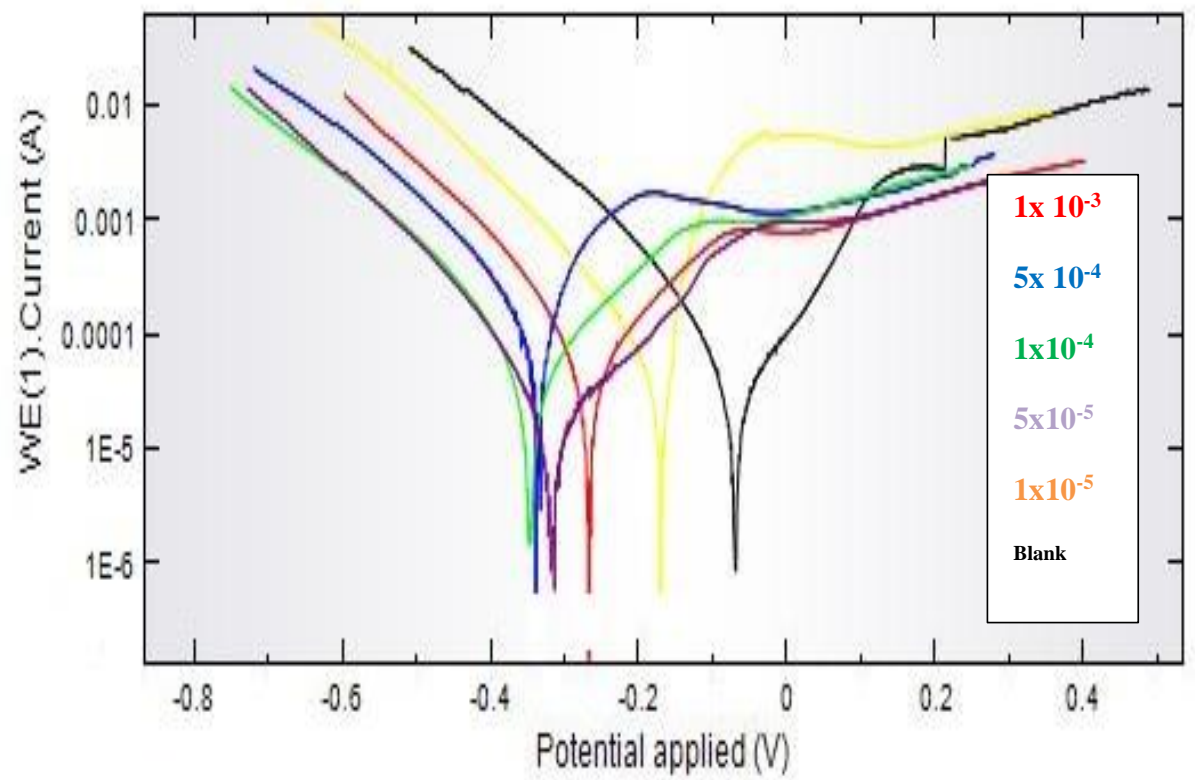

Fig (5) Polarization curves of carbon steel in $1.00 \mathrm{M} \mathrm{HCl}$ in absence and presence of various concentrations of compound [I]. 
Table (5) Parameters of carbon steel corrosion in free and inhibited 1.0 M HCl solutions as revealed from polarization technique.

\begin{tabular}{|c|c|c|c|c|c|}
\hline $\begin{array}{l}\text { Conc. } \\
\text { M }\end{array}$ & $\begin{array}{l}-\mathbf{E}_{\text {corr }} \\
\mathbf{m V}\end{array}$ & $\begin{array}{c}\beta_{a} \\
\text { mV/decade }\end{array}$ & $\begin{array}{c}-\boldsymbol{\beta}_{\mathrm{c}} \\
\mathrm{mV} / \mathrm{decade}\end{array}$ & $\begin{array}{c}I_{\text {corr }} \\
\mu \mathrm{A} / \mathbf{c m}^{2}\end{array}$ & IE\% \\
\hline 0 & 76 & 45 & 84 & 0.9 & ----- \\
\hline $1 \times 10^{-5}$ & 96 & 53 & 66 & 3.88 & -331 \\
\hline $5 \times 10^{-5}$ & 99 & 55 & 59 & 3.20 & -256 \\
\hline $1 \times 10^{-4}$ & 105 & 62 & 55 & 1.20 & -3 \\
\hline $5 \times 10^{-4}$ & 112 & 65 & 48 & 0.17 & 80 \\
\hline $1 \times 10^{-3}$ & 120 & 70 & 45 & 0.16 & 82 \\
\hline
\end{tabular}

Electrochemical impedance spectroscopy

The electrochemical behavior of carbon steel in the presence and absence of the additive compound was investigated by electrochemical impedance technique. The obtain Nyquist plots are shown in Fig (6). The Nyquist spectra attained by carbon steel in free and inhibited acid solutions were single conductive loop for all concentrations. The width of the Nyquist plot loop is smaller for low additive concentrations and higher for high additive concentrations than that of the free acid. Therefore, the additive compound acts as corrosive agent at its loser concentration while it acts as corrosion inhibitor at its higher concentrations.
This finding confirms the conclusion obtained by weight loss and Tafel polarization techniques. Table (6) contains the impedance parameters calculated from Nyquist spectra. The data of the table show that the charge transfer resistance value decreases in low additive concentrations and increases at higher ones. The inhibition efficiency values show some discrepancy from those obtained from weight loss and polarization techniques but has the same trend of variation with additive concentration. Such discrepancy could be attributed to the difference of the nature of these techniques.

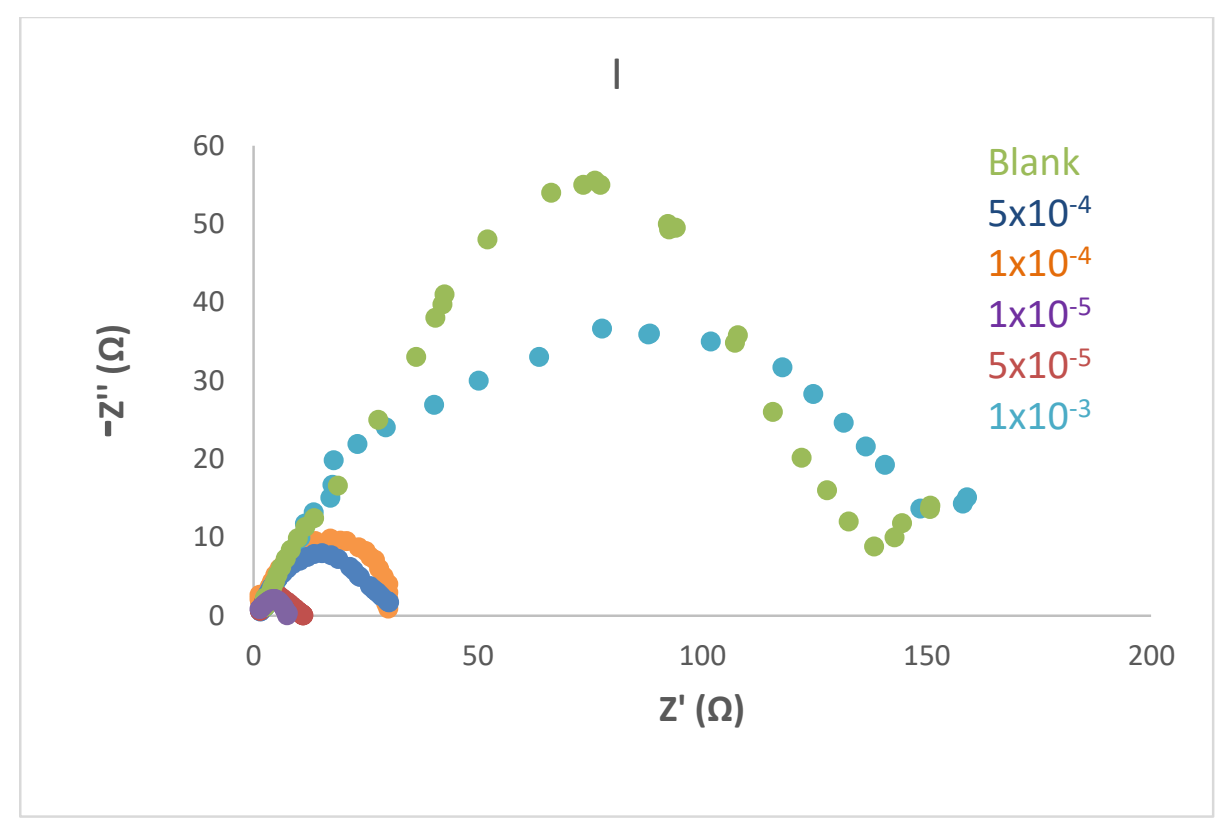

Fig (6) Nyquist diagrams of carbon steel in $1 \mathrm{M} \mathrm{HCl}$ solutions devoid of and containing different concentrations of compound [I]. 
Table (6) Impedance parameters for Carbon steel corrosion in 1M HCl solutions devoid of and containing different concentrations of compound [I].

\begin{tabular}{cccc}
\hline Conc. & $\mathbf{R}_{\mathbf{s}}$ & $\mathbf{R}_{\text {ct }}$ & \% IE \\
\hline Blank & 0.9552 & 31.478 & - \\
$\mathbf{1 x 1 0}$ & 1.4000 & 46.000 & 31.56 \\
$\mathbf{5 x 1 0}^{-5}$ & 1.0493 & 57.624 & 45.37 \\
$\mathbf{1 x 1 0}^{-4}$ & 1.0575 & 58.561 & 46.24 \\
$\mathbf{5 x 1 0}^{-4}$ & 1.1116 & 68.976 & 54.36 \\
$\mathbf{1 x 1 0}^{-3}$ & 1.1368 & 71.235 & 55.80 \\
\hline
\end{tabular}

\section{References}

[1] G.Y. Nagesh, K.M. Raj, B.H.M. Mruthyunjayaswamy, J.,Molecular Structure. Vol. 1079, PP. 423-432, 2015.

[2] S. Shukla, R.S. Srivastava, S.K. Shrivastava, A. Sodhi, Medical Chemistry Research. Vol.22, PP.1604-1617, 2013.

[3] M. Salehi, A. Amoozadeh, A. Salamatmanesh, M. Kubicki, J.,Molecular Structure. Vol.1091, PP.81-87, 2015.

[4] R. Miri, N. Razzaghi-asl, M. K. Mohammadi, J.,Molecular Modeling.Vol.19, PP. 727-735, 2013.

[5] S. M. M. Ali, M. Abul Kalam Azad, M. Jesmin, M. K. Islam, J.,Tropical Biomedicine, Vol.2, PP.438-442, 2012.

[6] D. Wei, N. Li, G. Lu, K. Yao, Science in China B, Vol.49, PP.225-229, 2006.

[7] A. Iqbal, H.L. Siddiqui, C.M. Ashraf, M. Ahmad,Molecules. Vol.12, PP.245-254, 2007.

[8] R.K. Agarwal, D. Sharma, L. Sing, Bioinorganic Chemistry and Applications.Vol. 1,PP. 1-9, 2006.

[9] E.M. Zayed, M.A. Zayed, Spectrochimica Acta Part A: Molecular Biomolecular and Spectroscopy. Vol.143, PP.81-90, 2015.

[10] E. Yousif, A. Majeed, K. Al-Sammarrae, Arabian J.,Chemistry ,Vol.10,PP. S1639S1644, 2017.

[11] A. Iqbal, H.L. Siddiqui, C.M. Ashraf, M. Ahmad, Molecules. Vol.12, PP.245-254, 2007.

[12] D. T. Tayade1 , S. P. Ingole, European J.,Pharmaceutical and Medical Research.Vol.3, PP.406-409, 2016.

[13] K. Mounika, B. Anupama, J. Pragathi, J.,Scientifc Research. Vol.2, PP.513-524, 2010.

[14] P. Venkatesh, Asian J.,Pharmaceutical and Health Sciences.Vol. 1, PP.8-11, 2011.
[15] Z. Cimerman, S. Miljanić, and N. Galić, "Croatica Chemica Acta.Vol.73, PP.81-95, 2000.

[16] M. A. Ashraf, K. Mahmood, A. Wajid, International Conference on Chemistry and Chemical Process IPCBEE.Vol.10, PP.1-7, 2011.

[17]H. Schiff, Justus Liebigs Annalen Der Chemie.Vol.131, PP.118-119, 1864.

[18] D. N. Dhar ,C. L. Taploo, J.,Scientific and Industrial Research.Vol. 41,PP. 501-506, 1982.

[19] J. Liu, B. Wu, B. Zhang, Y. Liu, Turk. J. Chem. Vol.30, PP.41-48, 2006.

[20] A. Budakoti, M. Abid, A. Azam, European J.,Medicinal Chemistry.Vol.41, PP.63-70, 2006.

[21] A. Kajal, S. Bala, S. Kamboj, N. Sharma, J.,Catalysts.Vol.49, PP.1-14, 2013.

[22] J. Kumar, A. Rai ,V Raj, Organic and Medicinal Chemistry International Journal. Vol. 1,PP. 217-224, 2017.

[23] N. Raman, and A. Selvan, Russian J.,Inorganic Chemistry.Vol.56, PP.759-770, 2011.

[24] P. Pfeiffer, E. Breith, E. Llibbe and T. Tsumaki, Justus Liebigs Ann. Chem.Vol. 503,PP. 84, 1933.

[25] G.C. Perryand , D.A.Thornton; J.,Inorganic and Nuclear Chemistry.Vol. 34, PP.3357, 1972.

[26] M. Arockia doss, S. Savithiri, G. Rajarajan, V. Thanikachalam, Spectrochimica Acta Part A: Molecular Biomolecular and Spectroscopy. Vol.148, PP.189-202, 2015.

[27] M. Arockia doss, S. Savithiri, G. Rajarajan, V. Thanikachalam, Spectrochimica Acta Part A: Molecular Biomolecular and Spectroscopy. vol .151,PP. 773-784, 2015.

[28] K. Gokula Krishnan, R. Sivakumar, V. Thanikachalam, H. Saleem, Spectrochimica 
Acta Part A: Molecular Biomolecular and Spectroscopy.Vol. 144, PP.29-42,2015.

[29] S. Savithiri, M. Arockia doss, G. Rajarajan, V. Thanikachalam, Spectrochimica Acta Part A: Molecular Biomolecular and Spectroscopy. Vol. 136,PP. 782-792, 2015.

[30] M. Murmu, S. Kr, N. Chandra, and P. Banerjee, Corros. Sci. Vol.146, PP. 134-151, 2019.

[31] H. Aouniti, S. Elmsellem, M. Tighadouini, S. Elazzouzi. Vol. 10, 2016.

[32] L.i Meena, P. Choudhary, A. Varshney, S. Varshney, Inter Jour of ChemTech Research.Vol.11, PP.337-346, 2018.

[33] N. Saxena, S. Kumar, M. K. Sharma, S. P. Mathur,Vol.15, PP.61-67, 2013.

[34] B. Delley, Phys. Rev.B:Condens. Matter. Vol.66, PP.155-125, 2002.

[35] in: Materials Studio, Accelrys software Inc.San Diego, USA Vol.18, PP.175-135, 2011.

[36] W. J. Hehre, Ab initio molecular orbital theory, Wiley-Interscience. Vol.68, PP.155125,1986 .

[37] H. Looker; I. Org. Chem,Vol. 27 PP. 361, 1962.
[38] Y. M. Issa, M. E. Moustafa, W. F. El-Hawary , M. Refaat, J. Ind. Chem. Soc., vol .74, PP.777-780, 1997.

[39] M. El-Kersh, M. Gaber, R. M. Issa I. M. Mansour, Kolor. Ert.Vol.6,PP.205, 1983.

[40] H. A. Dessouki, R. M. Issa , M. E. Moustafa; spectrochim. Acta. Vol. [A], PP.45, 775, 1989.

[41] S. M. Ibrahem, Ph. D. Thesis, Chem. Dep , Faculty of Sci., Benha Univ. Vol.6,PP.205, 1983. 2017.

[42] N. Benarous, A. Cherouana, E. Aubert, P. Durand, J. Mol. Struct.,Vol. PP.1105,186, 2016.

[43] E. Elamurugu Porchelvi, and S. Muthu , Spectrochimica Acta Part A: Molecular Spectroscopy.Vol. 134, PP.453-464, 2015.

[44] G.D. Tanga, J.Y. Zhao, R.Q. Li, Y.Cao, Z.C, Zhang, Spectrochim. Acta Part A. Vol.78, PP. 449, 2011.

[45] T.A. Yousef, O.A. El-Gammal, Sara F. Ahmed, G.M. Abu El-Reash, Spectrochimica Acta Part A: Molecular and Biomolecular Spectroscopy. Vol. 135,PP. 690-703, 2015.

[46] R. R. Ternavisk, A. J. Camargo, F. B.C. Machado, J. A.F.F. Rocco, J.,Molecular Modeling. Vol. 20, PP.2526-2528, 2014. 